\title{
Attribute Analysis for Bangla Words for Universal Networking Language(UNL)
}

\author{
Aloke Kumar Saha \\ Dept. of Computer Science \& Engineering \\ University of Asia Pacific \\ Dhaka, Bangladesh \\ Shammi Akhtar \\ Dept. of Computer Science \& Engineering \\ University of Asia Pacific \\ Dhaka, Bangladesh
}

\author{
Muhammad Firoz Mridha \\ Dept. of Computer Science \& Engineering \\ University of Asia Pacific \\ Dhaka, Bangladesh \\ Jugal Krishna Das \\ Dept. of Computer Science and Engineering \\ Jahangirnagar University \\ Savar, Dhaka, Bangladesh
}

\begin{abstract}
The Universal Networking Language (UNL) is an artificial worldwide generalizes form human interactive in machine independent digital platform for defining, recapitulating, amending, storing and dissipating knowledge or information among people of different affiliations. The theoretical and practical research associated with these interdisciplinary endeavor facilities in a number of practical applications in most domains of human activities such as creating globalization trends of market or geopolitical independence among nations. In our research work we have tried to develop analysis rules for Bangla part of speech which will help to create a doorway for converting the Bangla language to UNL and vice versa and overcome the barrier between Bangla to other Languages.
\end{abstract}

Keywords-Universal Networking Language; morphology; Bangla part of speech; morphological rules.

\section{INTRODUCTION}

Today the regional economics, societies, cultures and education are integrated through a globe-spanning network of communication and trade. This globalization trend evokes for a homogeneous platform so that each number of the platform can apprehend what other intimates and perpetuates the discussion in a mellifluous way. However the barriers of languages throughout the world are continuously obviating the whole world from congregating into a single domain of sharing knowledge and information. As a consequence United Nation University/Institute of advance Studies (UNU/IAS) were decided to develop an inter-language translation program. The corollary of their continuous research leads to a common form of language known as Universal Networking Language (UNL) [1].

The UNL acts as an intermediate form computer semantic language whereby any text written in a particular language is converted to a text of any other forms of language [2]. UNL, in other words is an artificial language for computer to express information and knowledge that can expressed in natural language. The rest of the paper is organized as the following. Section II outlines the UNL general structure.
In Section III Bangla part of speech and in section IV provides a Rule generation for Bangla part of speech for UNL expression.

\section{STRUCTURE OF UNL}

UNL system composed of three parts namely Universal words, Attributes labels and relational labels. Universal word (UW) which is actually nothing but English like word and is represented by nodes in a hyper graph $[1,7]$. Nodes associated with a sentence are connected by a relation known as symbolic relation. Each UW has some attributes that uniquely specifies that word and is placed according to a conceptual hierarchy derives from a knowledge base. However each of the UWs is comprised of Headword along with some constraints. The headword is considered as the form of native language word known as label whereas each of constraints in a constraint list of the Universal word corresponds to a concept of that word. The attributes lists associated with the individual universal word are used to represent the subjectivity of word based on their grammatical properties $[5,6]$.

The knowledge base (KB) which actually holds every possible combination of semantic relations basically plays two roles. Firstly it defines semantics of UWs and then provides linguistics knowledge of concepts. The KB however not only provides linguistics knowledge in computer understandable format but also provides the semantics background of UNL expressions [8].

In addition to the above parts the UNL system has a language server which can be fragmented into two parts known as en-converter (EnCo) and de-converter (DeCo). The converter builds a framework, independent of the diversity of languages, for morphological, semantic analysis and converts the native language text into UNL expressions autonomously [14]. To perform the conversion operation the EnCo uses word dictionary, knowledge base and en-cnversion rules. In contrast the DeCo acts the reverse way the EnCo does [15]. The general formats of the word dictionary entry are defined by UNL as follows:

[HW] "UW" (ATTRIBUTE1, ATTRIBUTE2 ...) < FLG, FRE, PRI> 
$\mathrm{HW} \leftarrow$ Head Word (Bangla Word)

$\mathrm{UW} \leftarrow$ Universal Word

ATTRIBUTE $\leftarrow$ Attribute of the HW

FLG $\leftarrow$ Language Flag

FRE $\leftarrow$ Frequency of Head Word

PRI $\leftarrow$ Priority of Head Word

\section{MORPHOLOGICAL ANALYSIS OF BANGLA PART OF} SPEECH

Morphology is the field of linguistics that studies the structure of words. It focuses on the patterns of word formation within and across languages, and attempts to formulate rules that model the knowledge of speakers of those languages. In natural language processing (NLP) we need to identify words in texts in order to determine their syntactic and semantic properties $[10,11]$. In the following section we are analyzing morphologically the different Bangla part of speech so that we can develop efficient rules for UNL expression.

\section{A. Grammatical Construction of words}

In this section, we have pointed out some essential grammatical issues about different parts of speech of Bangla that must be needed for English to Bangla MT dictionary.

\section{B. Parts of speech}

In Bangla language word may be categorized in one of five categories: noun, pronoun, adjective, verb and indeclinable [16]. Here, adverb is considered as adjective and the type indeclinable is concerned as preposition, conjunction and interjection.

Noun Morphology: Bangla Nouns have very strong and structural inflectional morphology base on case. Case of noun may be nominative ("ছেলে", boy), accusative (ছেলে-কে"), to the boy) and genitive (“জেলে-র”, of the boy) and so on. Gender and number are also important for identifying proper categories of nouns. Number may be singular ("জেলে", boy or "ছেলেটি", the boy, “বই”, book, “বইটি", the book) plural ("ছেলেরা", boys "ছেলেগুলি", the boys "বইগুলো", the books etc.). So, from the word "ছেলে" we get "ছেলের", "ছেলেকে", " ছেলেটি", "ছেলেগুলি" etc. and from the word "বই" we get “বইটি”, “বইগুলি” etc. Some dictionary entries may look like.

\section{[জেলে $]\{\}$ "boy (icl>person)" (N, HN, C, ANI) $<\mathrm{B}, 0,0>$}

Here, "boy (icl>person)" is the UW for "েেলে" but “র”, "কে" etc. have no UWs. Therefore, they should be represented in the dictionary only with grammatical attributes as follows.

$$
\begin{aligned}
& \text { [রা] }\{\} \text { “" (3P, PL, SUF, N, HUMN) <B, } 0,0> \\
& \text { [টি] }\{\} \text { “" (N, SG, SUF,3P) <B,0,0> } \\
& \text { [গুলি] }\{\} \text { “’”(N, PL, SUF,3P) }(<\mathrm{B}, 0,0> \\
& \text { [গুলো] }\{\} \text { “’”(N, SG, SUF,3P) <B,0,0> }
\end{aligned}
$$

We use 3P, SUF and $\mathrm{N}$ as grammatical attributes with “রা", because “রা” is used with third person say “ছেলেরা", N for noun and SUF as “রা" is a suffix. We have to put meticulous attention while defining the grammatical attributes. Because we use HUMN for human noun as “কে”, “রা" are used with human being only, say ছেলেকে, তাহাকে, but not গরকে,গরুরা etc. But we can not use HUMN with “র”, “টি”, “„গুলি” and "„গুলো” because they are used with both human and non human, say পাথির, ছেলেটি, গরুগুলো, etc.

Pronoun Morphology: There are eight different types of pronouns in English language. In Bangla language, there are ten types. They are: (i) Personal (I - Avwg (pronounce as “Ami”)), (ii) Reflexive (Myself $-{ }^{-} \wedge \mathrm{qs}$ ), (iii) Near indicating demonstrative (This - GB), (iv) Far indicating demonstrative (That $-\mathrm{H}$ ), (v) Collective (All $-\mathrm{mKj}$ (pronounce as "Sakal")), (vi) Interrogative (What - wK), (vii) Indefinite (Some - wKQy), (viii) Reciprocal (Each other - ci úi), (ix) Relative (Who - whwb) and (x) Others indicating (Other $\left.A b^{\prime}\right)$. In English language, near indicating and far indicating demonstrative pronoun is collectively defined as demonstrative pronoun. Collective and others indicating pronouns are concerned as indefinite pronoun. Here we can consider the word root “তাহা”'(he/she). From this we get তাহারা, তাহা-কে, তাহা-দের, তাহা-দের-কে, তাহা-দিগকে etc. So, we have to consider these morphemes রা, কে, দের, দিগকে for dictionary entries to form words with “তাহা" as above.

Adjective Morphology: Adjectives are of four types: proper adjective (evsjvł kx Kvco (pronounce as "Bangladeshi Kapor")), adjective of quality (mys i dzj (pronounce as "Sundor Ful")), adjective of quantity (w $\left.\varnothing_{s} b\right)$,

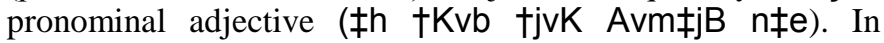
English, there are eight types of adverbial adjectives; but in Bangla, there are four categories. They are: adjective that modifies a verb (ax $\ddagger \mathrm{hvl}$ ), adjective that modifies another adjective (Lye fvj †jvK (pronounce as "Khub Valo Loke")), adjective that modifies an indeclinable (Avwg †Zvgvi gZ 'a" $Z$ Pwj), adjective that modifies a sentence (avwg@K †jv $\ddagger$ KivB cÖK,Z myLx). As Adjective we can consider Bangla words “সাহস", "সুন্দর" and "ভাল" meaning "bravery", "beautiful" and "good" in English respectively. From the first word we get সাহসী (সাহস+ই), সাহসের (সাহস+এর). And from the second and third words we get সুন্দরী, ভালোর, ভালোটা etc. We have to have the dictionary entries for সাহস, সুন্দর, ভালো, ই, এর, র, টা to make the meaningful words সাহসী, সাহসের, সুন্দরী, ভালোটা etc. by combining the morphemes with the root words using analysis rules.

Verb Morphology: Verbs, one of the most important word categories for dictionary design, may be classified in six categories. They are: (i) Intransitive verb ( $¥$ Q $j j i v ~ \ddagger L j \ddagger Q$ (pronounce as "Chelera Khelche")), (ii) Transitive verb ( $¥ \mathbb{Q} \ddagger j i v$ ej $\dagger L j \ddagger Q$ ), (iii) Di-transitive verb (evev Avgv $\ddagger K$ GKwU Kjg w $\ddagger q \ddagger Q b)$, (iv) Causative verb(gv wkï KK Pvu` † Lvb), (v) Compound verb (a nonfinite verb + a finite verb, NUbvwU ïłb ivL) and (vi) Complex verb (a noun/adjective/resounding indeclinable + verb, ZvRgnj `k@b 
Kijvg (pronounce as “Tajmohol Dorshon Korlam”)). In verb, mood is also an important feature. There are four types: (i) Indicative Mood (Avwg eB cwo), (ii) Imperative Mood (gb w`q co), (iii) Subjunctive Mood (cołj cvm Kiłe), and (iv) Optative Mood (Zvi g1/2j †nvK). We can give the example of the Bangla word "Бল" (means go). The corresponding UW in basic form is "go". The dictionary entry is: [ठলा] \{\} "go (icl>do)", where 'চলा\&' is the head word and (icl>do) is from the knowledge base. Some possible transformations of "চল" in the Bangla to UNL dictionary are given as follows [9, 10].

If we consider 'চল' (means go) as a root, we can represent this root in the dictionary as

[ठল] \{\} “go (icl>do)”(V,@present)<B,0,0>

Some transformations based on the persons and tenses are.

For first person:

[চল] \{\} “go (icl>do)" (ROOT, BANJANT)<B, 0, 0>

[ই] \{\} "go (icl>do)" (ROOT, BANJANT, PRESENT INDEF) $<\mathrm{B}, 0,0>$

[ইতেছি] \{ \} “go (icl>do)" (ROOT, BANJANT, PRESENT CONT) $<\mathrm{B}, 0,0>$

For second person:

[চল] \{\} “go (icl>do)" (ROOT, BANJANT)<B, 0, 0>

[ইতেছ্] \{ \} "go (icl>do)" (ROOT, BANJANT, PRESENT CONT) $\langle\mathrm{B}, 0,0\rangle$

[ইবে] \{ \} "go (icl>do)" (ROOT, BANJANT, FUTURE INDEF) $<\mathrm{B}, 0,0>$

For third person:

[চল] \{\} “go (icl>do)" (ROOT, BANJANT)<B, 0, 0>

[ইয়াছ্ছে] \{\} "go (icl>do)" (ROOT, BANJANT, PRESENT PERF) $<\mathrm{B}, 0,0>$

[ইতেছে] \{\} “go (icl>do)" (ROOT, BANJANT, PRESENT CONT) $<\mathrm{B}, 0,0\rangle$

For resolving the ambiguities of the words গিয়েছ্ছি, গিয়েছ্ছিলাম, গিয়েছ্নেন, গিয়েছিলেন, যাইতে থাকবে, etc. we have to define them as full words for dictionary entries. For instance [গিয়েছ্লিলাম] \{\} “go (icl>move>do)"(V, PAST, INDEF, 1P). Using the same procedure we can make dictionary entries for different transformations of other roots such as কর (do), লিথ (write),দে (give) etc.

\section{Gender}

In both Bangla and English Language, Gender is classified into four groups. They are: (i) Masculine (Father - evev (pronounce as "Baba")), (ii) Feminine (Mother - gv (pronounce as "Ma")), (iii) Common (Human - gvbyl (pronounce as "Manush")) and (iv) Neuter (Book - eB (pronounce as "Boi")).

\section{Number}

Both Bangla and English language, there are two types of number. They are: (i) Singular number, and (ii) Plural number.

\section{RULES FOR UNL TEXT GENERATION}

In this section, we have presented some Bangla morphological rules for regular inflections, derivations and compounding with additional explicit rules for irregular inflection, derivation and compounding.

\section{A. Analysis Rules}

An analysis rule describes rule application conditions, a method to rewrite the attribute of node that satisfies the application condition, and construction methods of syntax tree. While applying rules, the EnConverter analyzes morphemes, syntax and semantics. Finally, it generates a syntax tree and a network. [11]:

The description format of the analysis rules is as follows

$$
\begin{aligned}
& \text { <TYPE> }
\end{aligned}
$$

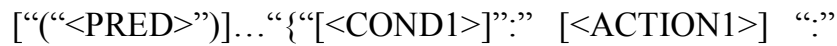

$$
\begin{aligned}
& {[<\text { ROLE1 }>\text { ] " }\} \text { " }} \\
& {[<\text { ROLE2 }>\text { ] " }\} \text { " }} \\
& \text { [“(“<SUF>")"] ..."P(“"<PRIORITY>”);" }
\end{aligned}
$$

\section{Symbol Explanation:}$$
\text { “'” }
$$

\{ $\}$ and () designates an analysis windows in the node list.

\section{Description of Condition:}

$<$ PRE $>$ Describes condition of nodes on the left side of the left of analysis window.

$<$ SUF> Describes condition of nodes on the right side of the right of analysis window.

$<$ COND1 $>$ Describes condition of the node in the Left Analysis Window (LAW).

$<$ COND2 $>$ Describes condition of the node in the Right Analysis Window (RAW).

\section{Description of Action:}

$<$ ACTION1> Describe the rewriting of grammatical attribute in the LAW.

$<$ ACTION2> Describe the rewriting of grammatical attribute in the RAW.

\section{Direction of Semantic Relation:}

It describes the semantic relation between the left node (LN) and the right node (RN). 
$<$ RELATION1 $>$ Describe the semantic relation of the RAW to LAW.

$<$ RELATION2>Describe the semantic relation of the LAW to RAW.

$<$ PRIORITY> Describes priority of the rules. Code 0-255 is used to specify the priority.

\section{B. Types of the Analysis Rules}

This part explains the action and functions of the rule types that can lie described with <TYPE $>$ in analysis rules.

Left Composition " $+|+:+|+: \mathrm{c} \mid+: * "$

The RN is combined to LN to make one composition node. The syntax tree and the attribute having left node are inherited. When the RN attributes is inherited, “@” is put in the action column of the LN, the original two nodes are deleted from the node list. The composition node is inserted into the node list. After applying the rules, the composition node takes a position in the RAW.

Right Composition " - |-:+|-:c|-:* "

The LN is combined to RN to make one composition node. The composition node is inserted into the node list. After applying the rules, the composition node takes a position in the LAW.

Left Modification "<"

When the RN modifies LN, the RN is deleted from node list and the LN remains only. The node, which the $<$ RELATION $>$ is described, is the to-node and the other node is from-node.

Right Modification ">"

When the LN modifies RN, the $\mathrm{LN}$ is deleted from node list and the $\mathrm{RN}$ remains only.

\section{Left Shift " $L$ "}

Shift the analysis window to the left.

Right Shift " $R$ "

Shift the analysis window to the right.

Attribute Changing Rule ":”

This rule adds or deletes attributes from a particular node.

\section{Morphological rule Generation for Bangla Parts of Speech}

Bangla is a semantic language, and its basic characteristic is the rich morphology in which most of its words are derived from roots. Inflections and derivations are generated by changing vowels and insertion of consonants. Bangla sentences are characterized by a strong tendency for agreement between its constituents: between verb and noun, noun and objective, in matters of numbers, gender, definitiveness, case, person, etc.

These properties are expressed by a comprehensive system of affixation. To satisfy these grammatical properties, the generation rules are expected to be complex for handling the processing of generating grammatically correct Bangla sentences from UNL expression and structure A database system has been developed for the classification and features adding for each entry in the dictionary [3, 4]. The selected Bangla word is then classified to Noun or Verb or Particle. The relation mapping is implemented in the en-conversion rule.

Bangla parts of speech conversion rules are mainly for noun $\leftrightarrow$ adjective. Some conversion rules are also done for indeclinable $\leftrightarrow$ noun and indeclinable $\leftrightarrow$ adjective and there are some exceptions also.

From the analysis of Bangla Part of speech, gender and number, one can readily find that they agree right composition rule.

a) Right Composition Rule: (For Bangla noun $\leftrightarrow$ adjective)

- Rule 1: C \{NOUN:::\} \{ CASEMARKER : +ADJ,CASEMARKER:: $\}$

For noun $\leftrightarrow$ adjective, add ' $\mathrm{X}$ ' with the last character (AvMgb-AvMgbx).

- Rule 2: C \{NOUN::: $\}$ \{ CASEMARKER : +ADJ,CASEMARKER:: $\}$

For noun $\leftrightarrow$ adjective, add ' $w$ ' with the last character and after that, add ' $Z$ ' $\left.(A v b)^{\prime}-A v b w\right\rangle^{\prime} Z$, Av $\left.\ddagger g v^{`}-A v \ddagger g v w ` Z\right)$.

- Rule 3: C \{NOUN::: $\}$ \{ CASEMARKER : +ADJ,CASEMARKER:: $\}$

For noun $\leftrightarrow$ adjective, add ' $¥ U$ ' after the last character (SMov-SMov -U).

- Rule 4: C \{NOUN:::\} \{ CASEMARKER : +ADJ,CASEMARKER:: $\}$

For noun $\leftrightarrow$ adjective, add ' $x$ ' with the last character and after that, add ' $q$ '(bvUK - bvUKxq)

- Rule 5: C \{NOUN:::\} \{ CASEMARKER : +ADJ,CASEMARKER:: $\}$

For noun $\leftrightarrow$ adjective, if the last character is ' $b$ / $Y$ / $q$ ', then drop the last character and add ' $w$ ' with the previous character and after that, add ' $Z$ '(mvab mvwaZ, AvniY - AvnwiZ, cwiPq - cwiwPZ).

- Rule 6: C \{NOUN::: $\}$ \{ CASEMARKER : +ADJ,CASEMARKER::

For noun $\leftrightarrow$ adjective, add '.", after the last character (†fvM - $¥ f v M ")$.

- Rule 7: C \{NOUN:::\} \{ CASEMARKER : +ADJ,CASEMARKER:: $\}$

For noun $\leftrightarrow$ adjective, add ' $D$ ' with the last of the word (Xvj + D = Xvjy).

- Rule 8: C \{NOUN::: $\}$ \{ CASEMARKER : +ADJ,CASEMARKER:: 
For noun $\leftrightarrow$ adjective, add 'DK' at last ( $¥ \mathrm{cU}+\mathrm{DK}$ $=\ddagger c U z K)$.

- Rule 9: C \{NOUN:::\} \{ CASEMARKER : +ADJ,CASEMARKER:: $\}$

For noun $\leftrightarrow$ adjective, add 'Av ' at last $(e v N+A v=$ evNv).

- Rule 10: C \{NOUN:::\} \{CASEMARKER : +ADJ,-CASEMARKER::

For noun $\leftrightarrow$ adjective, add ' Bqv / D $\neq \mathrm{i}$ ' at last (kni + Bqv/Dłi = knwiqv/kûłi).

- Rule 11: C \{NOUN:::\} \{ CASEMARKER : +ADJ,CASEMARKER:: $\}$

For noun $\leftrightarrow$ adjective, add ' $† P$ ' at last (jvj $+\ddagger P$ $=j v j \ddagger P$ ).

- Rule 12: C \{NOUN:::\} \{CASEMARKER : +ADJ,-CASEMARKER:: $\}$

For noun $\leftrightarrow$ adjective, add ' $¥ Z \mathrm{~V}$ ' at last ( $\mathrm{dzcv}+\dagger Z \mathrm{v}$ $=\mathrm{dzcv} \ddagger Z \mathrm{v}$ ).

- Rule 13: C \{ ADJ :::\} \{ CASEMARKER : + NOUN ,- CASEMARKER::

For adjective $\leftrightarrow$ noun, add 'AvB ' at last ( $w g V v+$ $A v B=w g \vee v B$ ).

- Rule 14: C \{ ADJ ::: \} \{ CASEMARKER : + NOUN ,- CASEMARKER:: $\}$

For adjective $\leftrightarrow$ noun, add 'Bgv ' at last (bxj + Bgv =bxwjgv).

- Rule 15: C \{ ADJ :::\} \{ CASEMARKER : + NOUN ,- CASEMARKER:: $\}$

For adjective $\leftrightarrow$ noun, add 'cbv ' at last ( ' iš- + $\mathrm{cbv}=$ ' $\sim \mathrm{iš}-\mathrm{cbv})$.

- Rule 16: C \{ADJ:::\} \{CASEMARKER : +NOUN,CASEMARKER:: $\}$

For adjective $\leftrightarrow$ noun, add 'Avwg ' at last ( ' ó + Avwg = $\sim$ óvwg).

- Rule 17: C \{ADJ:::\} \{ CASEMARKER : +NOUN,CASEMARKER:: $\}$

For adjective $\leftrightarrow$ noun, add 'wMwi' at last (evey + wMwi =eveywMwi).

- Rule 18: C \{ADJ:::\} \{CASEMARKER : +NOUN,CASEMARKER:: $\}$

For adjective $\leftrightarrow$ noun, add ' $Z \mathrm{v}$ ' at last $(\mathrm{GK}+\mathrm{Zv}=$ GKZv).

- Rule19: C\{INDECLINABLE:::\} \{CASEMARKER: +NOUN,- CASEMARKER::
For indeclinable $\leftrightarrow$ noun, drop ' $v$ ' and add ' " , (Z_v -Z_").

\section{b) Rules for Gender}

In gender, a masculine or a feminine word is derived from another feminine or another masculine word. Here we have derived some morphological rules for the conversion of Bangla Gender:

- Rule 1: C \{ MALE :::\} \{CASEMARKER : +FEMALE,-CASEMARKER,+ CASEMARKER:: Dropping the last ' $v$ ' and adding the vowel ' $\mathrm{x}$ ', some masculine words are converted to feminine $(\mathrm{PvPv}-\mathrm{PvPx})$.

- Rule 2: C \{ MALE ::: \} \{CASEMARKER : +FEMALE,-CASEMARKER,+CASEMARKER::\} Adding ' $b x$ ', some masculine words are converted to feminine ( $\ddagger$ avcv - $\ddagger$ avcvbx).

- Rule 3: C \{ MALE ::: \} \{CASEMARKER : +FEMALE,- CASEMARKER,+ CASEMARKER:: If there is ' $x$ ' in the masculine word, then the ' $x$ ' is converted to ' $w$ ' and at the end, ' $b x$ ' is added (wfLvix - wfLvwibx).

- Rule 4: C \{ MALE :::\} \{CASEMARKER : +FEMALE,- CASEMARKER,+ CASEMARKER::\} Adding 'Avbx', some masculine words are converted to feminine (wng - wngvbx).

c) Rules for Number

Here are few rules for the translation of Bangla Number:

- Rule 1: C \{NOUN, SING:::\} \{NUMBERSIGN : + PLU, - NUMBERSIGN :: $\}$

Adding “ wU, Uv, Lvbv, Lvwb" with the main word represents singular word $\left(M^{\prime \prime}\right.$ - Mi"wU, evQyi evQyiUv, LvZv - LvZvLvbv, eB-eBLvwb).

- Rule 2: C \{NOUN,SING,HIGH::: \}

\{NUMBERSIGN : +PLU,- NUMBERSIGN ::\} Adding "iv, Giv, MY, e,, ', gÛjx, eM@” with the main word represents the high class living things in plural number (Qvî-Qvîiv, gv-gvłqiv, Rb-RbMY, wkףK - wkףKgÛjx .

- $\quad$ Rule 3: C \{NOUN,SING,LIVING::: $\}$ \{NUMBERSIGN : +PLU,- NUMBERSIGN ::\} Adding " Kzj, mKj, me, mg n " with the main word represents the low class living things and non-living things in plural number (Kwe-KweKzj, fvBfvBme).

- Rule 4: C \{NOUN, SING, NON-LIVING ::: $\}$ \{NUMBERSIGN: +PLU,- NUMBERSIGN :: Adding “ jjv, ,wj, , $\ddagger j v$, ,"Q, 'vg, ivwk, Avewj, wbKi, cyÄ, gvjv, ivwR, wbPq" with the main word represents the non-living things and dumb things in 
plural number (Avg-Avg jv,Kzmyg-Kzmyg`vg, evwj-evwjivwk, ZviKv-ZviKvivwR).

\section{CONCLUSION}

In this paper we have presented morphological rules for Bangla part of speech, number and gender. To do so we did morphological analysis of Bangla part of speech. We hope that these rules would be useful for conversion of Bangla sentence to UNL expressions and vice-versa.

Even though the limited numbers of rules are considered in this paper, it theoretically shows that the designed model works perfectly for Bangla words. All the Bangla words and rules will be considered in future.

\section{REFERENCES}

[1] H. Uchida, M. Zhu, "The Universal Networking Language (UNL) Specification Version 3.0", Technical Report, United Nations University, Tokyo, 1998

[2] Uchida H.,Zhu,M. and Della Senta, "A Gift for a Millennium. The United Nations University,Tokyo,Japa,2000”

[3] Muhammad Firoz Mridha, Manoj Banik, Md. Nawab Yousuf Ali, Mohammad Nurul Huda, Chowdhury Mofizur Rahman, Jugal Krishna Das, "Formation of Bangla Word Dictionary Compatible with UNL Structure", SKIMA 2010, August 25-27, Paro, Bhutan.

[4] Muhammad Firoz Mridha, Md. Zakir Hossain, Manoj Banik, Mohammad Nurul Huda, ChowdhuryMofizur Rahman, Jugal Krishna Das, "Development of Grammatical Attributes for Bangla Root and Primary Suffix for Universal Networking Language", SKIMA 2010, August 25-27, Paro, Bhutan.

[5] S. Abdel-Rahim, A.A. Libdeh, F. Sawalha, M. K. Odeh, "Universal Networking Language(UNL) a Means to Bridge the Digital Divide",
Computer Technology Training and Indistrial Studies Center, Royal Scientific Sciety, March 2002.

[6] M. M. Asaduzzaman, M. M. Ali, "Morphological Analysis of Bangla Words for Automatic Machine Translation", International Conference on Computer, and Information Technology (ICCIT), Dhaka, 2003, pp.271-276

[7] Serrasset Gilles, Boitel Christian, (1999) UNL-French Deconversion as Transfer \& Generation from an Interlingua with Possible Quality Enhancement through Offline Human Interaction. Machine Translation Summit-VII, Singapore.

[8] M. E. H. Choudhury, M. N.Y. Ali, M.Z.H. Sarkar, R. Ahsan, "Bridging Bangla to Universal Networking Language- A Human Language Neutral Meta- Language", International Conference on Computer and Information Technology (ICCIT), Dhaka, 2005,pp.104- 109

[9] M.E.H. Choudhury, M.N.Y. Ali, "Framework for synthesis of Universal Networking Language", East West University Journal, Vol. 1, No. 2, 2008, pp. 28-43

[10] M.N.Y. Ali, J.K. Das, S.M. Abdullah Al Mamun, M. E.H. Choudhury, "Specific Features of a Converter of Web Documents from Bengali to Universal Networking Language", International Conference on Computer and Communication Engineering 2008(ICCCE'08), Kuala Lumpur, Malaysia.pp. 726-731

[11] S. Dashgupta, N. Khan, D.S.H. Pavel, A.I. Sarkar, M. Khan, "Morphological Analysis of Inflecting Compound words in Bangla", International Conference on Computer, and Communication Engineering (ICCIT), Dhaka, 2005, pp. 110-117

[12] M.N.Y. Ali, J.K. Das, S.M. Abdullah Al Mamun, A. M. Nurannabi," Morpholoical Analysis of Bangla worfs for Universal Networking Language", icdim, 08 .

[13] Bangla Academy (2004), Bengali-English Dictionary, Dhaka.

[14] Enconverter Specifications, version 3.3, UNL Center/ UNDL Foundation, Tokyo, Japan 2002.

[15] Deconverter Specifications, version 2.7, UNL Center/ UNDL Foundation, Tokyo, Japan, 2002

[16] D. S. Rameswar, "Shadharan Vasha Biggan and Bangla Vasha", Pustok Biponi Prokashoni, November 1996, pp.35

[17] D. S. Rameswar, "Shadharan Vasha Biggan and Bangla Vasha", Pustok Biponi Prokashoni, November 1996, pp.35 